\title{
Hypothesis
}

\section{An analysis of phosphorylation sites in protein kinases from Leishmania}

\section{Sukrat Sinha \& Shanthy Sundaram}

Centre of Biotechnology, University of Allahabad, Allahabad, Uttar Pradesh - 211002 India; Sukrat Sinha - E-mail: sukratsinha@gmail.com

Received June 14, 2016; Revised July 4, 2016; Accepted July 5, 2016; Published July 26, 2016

\begin{abstract}
Protein kinases are promising drug targets for Leishmaniasis. We have evaluated the phosphorylation potential of protein kinases in different species and strains of Leishmania. Phosphorylation potential of serine, threonine and tyrosine residues of kinases in Leishmania parasite were studied. The results indicate that some species specific residues of serine, threonine and tyrosine have a phosphorylation potential of 1 suggesting that these residues are important target sites in protein kinases based anti-leishmanial therapies.
\end{abstract}

\section{Background:}

Leishmaniasis is an infectious disease complex caused by several species that are members of the protozoan parasite genus Leishmania. In humans, disease manifestation ranges from selfhealing cutaneous lesions to life threatening visceral leishmaniasis (VL). This disease complex affects 12 million people and there are 1.5 million new cases annually [1]. VL, caused by Leishmania donovoni, Leishmania infantum and Leishmania chagasi, remains the main agent of morbidity and mortality in leishmaniasis. The parasite has a simple life cycle, and abundant clinical and experimental evidence indicates that of all the parasitic diseases, leishmaniasis in particular should be an appropriate target for effective control through vaccination. There are, however, no vaccines in routine use against any form of the disease [1-2]. Currently available vaccines against a variety of infectious diseases mediate protection by a long-lived humoral response through the production of antibodies. For diseases such as tuberculosis, malaria, human immunodeficiency virus infection, and leishmaniasis, however, the cellular immune response comprising primarily Th1 and CD8 effector T cells has been shown to be critical for mediating protection against the infection. Kinase catalyses the transfer of phosphate groups from high-energy phosphate donating molecules to specific substrates. The process known as phosphorylation, where the substrate gains a phosphate group and

ISSN 0973-2063 (online) 0973-8894 (print)

Bioinformation 12(4): 249-253 (2016) the high-energy ATP molecule donates a phosphate group [3].

Protein kinases act on serine, threonine, tyrosine, or histidine residues of proteins leading to phosphorylation. Phosphorylation can modify the function of a protein in a variety of ways. It can alter activity of a protein, stabilize it or cause degradation, localize it within a particular compartment of a cell, can initiate or disrupt its interaction with other proteins. The protein kinases make up the majority of all kinases and are widely studied. These kinases along with phosphatases, play a major role in protein and enzyme regulation along with signaling in the cell [3].

Adaptation to changes in the environment is usually initiated by extracellular signals that regulate the transcription of specific genes [4]. The transduction of signals to the nucleus of eukaryotic cells is mainly mediated by phosphorylation cascades through the activity of serine/threonine and tyrosine kinases. The central role of protein phosphorylation in signal transduction, cellular transformation and differentiation in eukaryotic systems has become well established. Complex networks of protein phosphorylation/dephosphorylation represent important mechanisms in cellular regulation and transformation. Protein phosphorylation at tyrosine appears to be common signaling mechanism in eukaryotes [4]. As in higher eukaryotes, it is likely 


\section{BIOINFORMATION \\ Discovery at the interface of physical and biological sciences}

\section{Open access}

that protozoans control a variety of intracellular events through the reversible phosphorylation of proteins. Understanding of phosphorylation and dephosphorylation events are of considerable interest, but are relatively unexplored in Leishmania donovani. Lopes and colleagues have provided evidence of an infection-enhancing role of PKR in L. amazonensis infections. L. amazonensis infection of cultured human or mouse macrophage cell lines induced the activation of PKR as monitored by the time-dependent increase in the phosphorylated form of PKR (pPKR) [5]. In cells infected by pathogens, there is an increasing appreciation that dysregulated expression of protein kinases promotes the success of intracellular infections. In the current study we have deduced the phosphorylation potential of serine, threonine and tyrosine residues of protein kinases of different species and strains of Leishmania.

\section{Methodology:}

Dataset creation

In this study, we aligned the protein sequences of Protein kinase of Leishmania spp associated with Leishmaniasis under similar prediction conditions and compared the results obtained. The protein sequences were procured from the National Center for Biotechnology Information (NCBI) through their Entrez search.

Table 1: A dataset of protein kinases from Leishmania species

\begin{tabular}{llllll}
\hline S. No & Accession no & Species name & \multicolumn{3}{c}{ Number of residues } \\
\cline { 4 - 6 } & & & Ser & Thr & Tyr \\
\hline 1 & gi_146085061 & Leishmania infantum JPCM5 & 24 & 14 & 12 \\
2 & gi_134069258 & Leishmania infantum JPCM5 & 10 & 3 & 4 \\
3 & gi_322498592 & Leishmania donovani & 11 & 3 & 4 \\
4 & gi_389603714 & Leishmania braziliensis MHOM/BR/ 75/M2904 & 10 & 3 & 4 \\
5 & gi_401421208 & Leishmania mexicana MHOM/GT/2001/U1103 & 11 & 5 & 4 \\
6 & gi_68126239 & Leishmania major strain Friedlin & 10 & 2 & 4 \\
7 & gi_685203703 & Leishmania panamensis & 14 & 3 & 4 \\
8 & gi_731695478 & Leishmania panamensis & 14 & 3 & 4 \\
9 & gi_731707594 & Leishmania panamensis & 24 & 10 & 6 \\
10 & gi_7658170 & Leishmania major & 4 & 0 & 0 \\
\hline
\end{tabular}

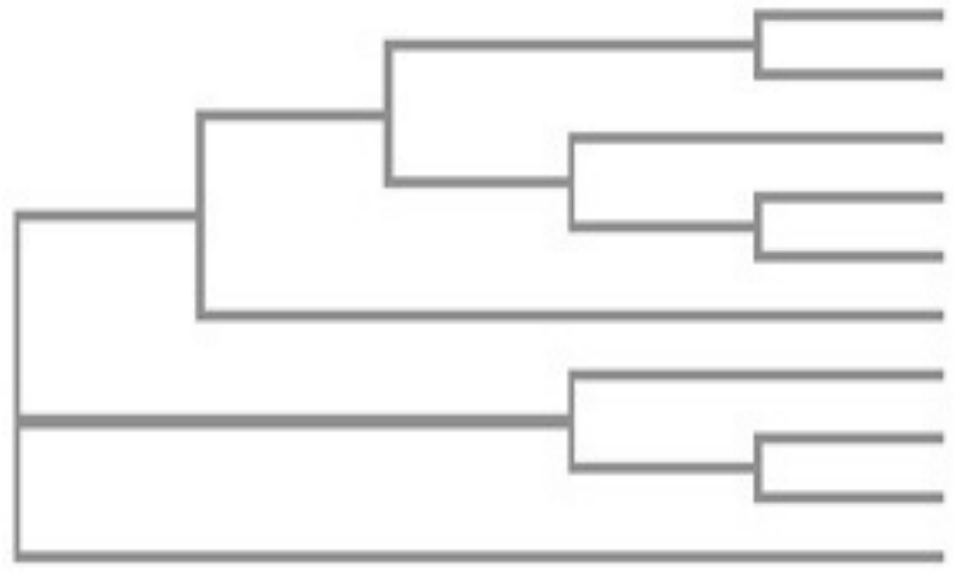

gi|731707594|ref|XP_010702617.1| 0.41331 gi|146085061|ref|XP_001465161.1| 0.53372 gi|389603714|ref|XP_003723002.1| 0.00241 gi|685203703|gb|AIN97944.1| 0 gi|731695473|ref|XP_010698651.1| 0 gi|7658170|gb|AAF66DS1.1| 0.03142 gi|401421203|ref|XP_003875093.1| 0.02931 gi|134069253|emj|CAM67408.1| 0.00191 gi|322498592|emb|CBZ33664.1| 0.0003 gi|68126239|emb|CA03611.1| 0.01551

Figure 1: Phylogenetic analysis of different species strains of protein kinase of Leishmania associated with Leishmaniasis. gi |731707594| and gi|146085061| are closely related. gi| 389603714|, gi|685203703| and gi|731695473| are closely related. Similarly gi|322498592|, gi $\mid 134069253$ | are closely related as compared to gi |401421203|. gi |68126239| is the most distant of all followed by gi | 7658170 |

ISSN 0973-2063 (online) 0973-8894 (print)

\section{BIOMEDICAL}

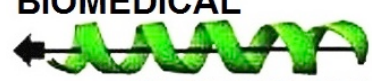

(C)2016 


\section{BIOINFORMATION}

Discovery at the interface of physical and biological sciences

\section{Open access}
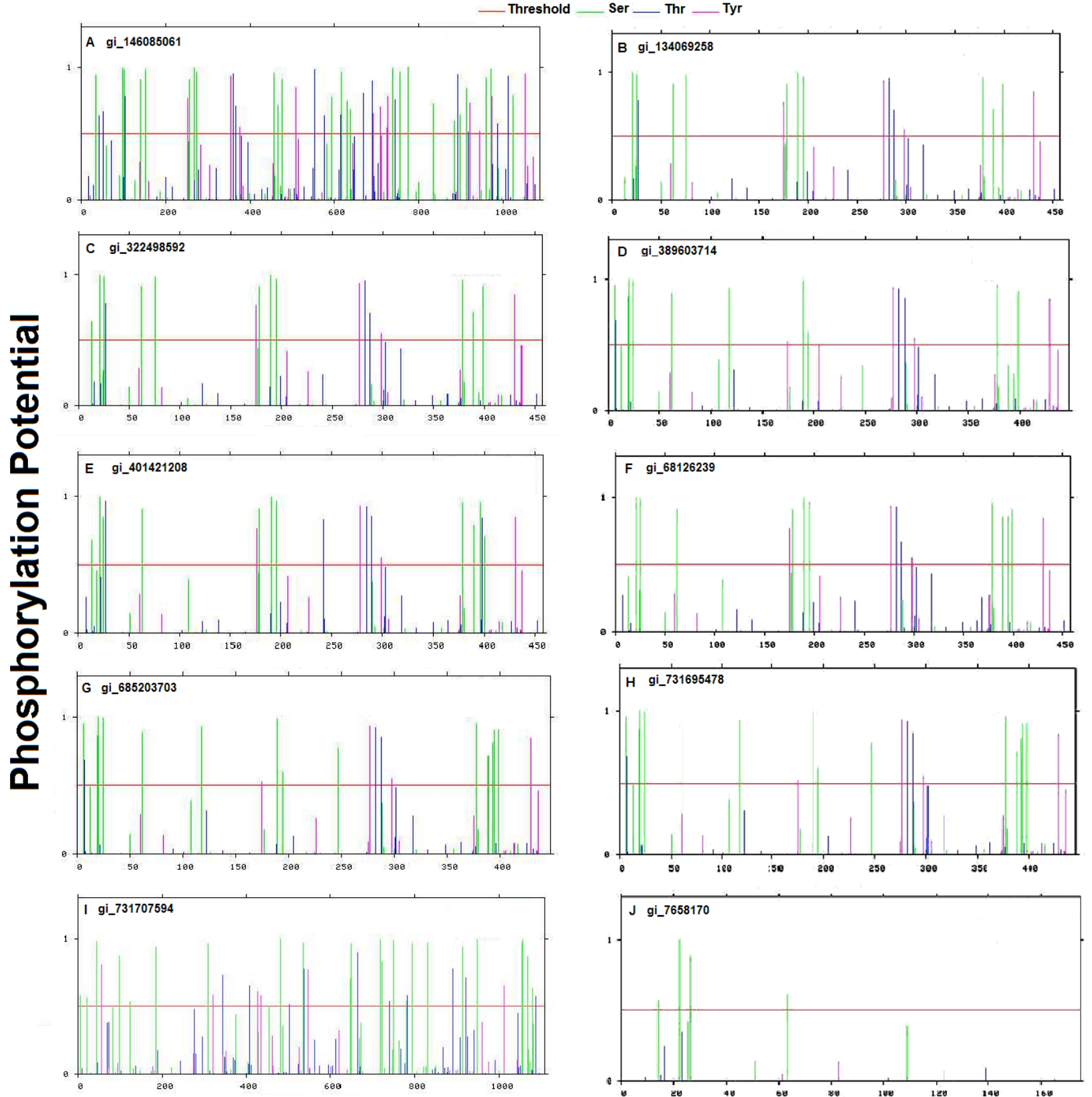

Sequence Position

Figure 2: A measure of phosphorylation potential among residues of kinases in different species of Leishmania (see Table 1 for further details).

Dendrogram

ISSN 0973-2063 (online) 0973-8894 (print)

BIOMEDICAL

Bioinformation 12(4): 249-253 (2016)

(C)2016

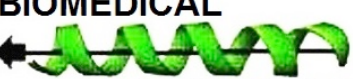

INFORMATICS 


\section{Open access}

A dendrogram (from Greek dendron "tree", -gramma"drawing") is a tree diagram used to illustrate the arrangement of the clusters produced by a clustering algorithm.

\section{Phylogeny}

A phylogenetic tree or evolutionary tree shows the evolutionary relationships among various biological species or other entities that are believed to have a common ancestor. In a phylogenetic tree, each node with descendants represented the most recent common ancestor of the descendants, and the edge lengths in some trees correspond to time estimates. We referred each node as a taxonomic unit. Internal nodes are generally called hypothetical taxonomic units (HTUs) as they cannot be directly observed.

\section{Phosphorylation potential}

A number of in silico tools have been developed in order to predict plausible phosphorylation sites in a given protein, lipid, carbohydrate, DNA or RNA. An attempt in this regard was made by [6] whereby they conducted a benchmark study including the leading protein phosphorylation prediction software, in an effort to determine which performs best. NetPhos 2.0 predicted all phosphorylation sites with fidelity of $69 \%$. So we performed prediction of phosphorylation sites using Net Phos 2.0.

Protein sequences of various species and strains expressing protein kinases having different accession nos were obtained from NCBI and further subjected to analysis of phosphorylation sites using Net Phos 2.0 input sequences must be in the one-letter code. Peptides shorter than 9 residues can produce non-reliable results. So it's always advisable to include 4 residues on both sides of the Tyr/Ser/Thr to be evaluated. The optimal is to use the complete "native" protein sequence. By default the server uses protein kinase specificity prediction without any filtering.

NetPhos is a neural network-based method for predicting potential phosphorylation sites at serine, threonine or tyrosine residues in protein sequences. The first version of NetPhos (v.1.0) was developed in 1997-1998 and was only available for in-house use and research collaborations. Version 2.0 was trained on a larger data set of known phosphorylation sites and has been made publicly available using the WWW or via e-mail. Protein phosphorylation at serine, threonine or tyrosine residues affect a multitude of cellular signaling processes. Here we study specificity in substrate recognition and phosphorylation by protein kinases. Net Phos is an artificial neural network method that predicts phosphorylation sites in independent sequences with sensitivity in the range from $69 \%$ to $96 \%$.

\section{Discussion:}

Leishmania activated Kinases (LACK) play an important role in parasite thermo tolerance and virulence [7]. Stage specific protein ISSN 0973-2063 (online) 0973-8894 (print) phosphorylation patterns [8] and an extensive examination of phosphorylation changes during promastigote-to-amastigote differentiation [9] have been recently described. Phylogeny analysis (Figure 1) shows evolutionary relationship of different species strains of protein kinase of Leishmania associated with Leishmaniasis. gi |731707594| and gi|146085061| are closely related. gi| 389603714|, gi|685203703| and gi|731695473| are closely related. Similarly gi|322498592 |, gi |134069253 | are closely related as compared to gi $|401421203|$. gi $|68126239|$ is the most distant of all followed by gi | 7658170|. Protein kinases gi_14608506 and gi_73170759 show higher number of serine and threonine residues while no of tyrosine residues are higher in the former strain. While the other strains like gi_13406925, gi_32249859, gi_38960371, gi_40142120, gi_68126239, gi_68520370, gi_73169547 have reasonable higher no of serine, threonine and tyrosines. gi_7658170 has only 4 residues of serine without any threonine and tyrosines which indicates lower potential of phosphorylation. In gi_14608506 (Figure 2A) potential of phosphorylation of serine is found to be highest at positions 35 and 268 with values 0.990 and 0.991 respectively, in threonine 554 and 661 with phosphorylation potential 0.677 and 0.647 respectively, tyrosine 355 and 1053 with phosphorylation potential 0.933 and 0.947 respectively, In gi_13406925 (Figure 2B) potential of phosphorylation of serine is found to be highest at amino acid residue position 75 and 190 with phosphorylation potential of 0.980 and 0.991 respectively, threonine shows high phosphorylation potential of 0.947 at position 283. In gi_32249859 (Figure 2C) serine showed phosphorylation potential of 0.990 at amino acid residue position 21 , threonine showed max phosphorylation potential of 0.947 at 283, tyrosine showed a phosphorylation potential of 0.932 at position 0.277. In gi_38960371 (Figure 2D) serine at the position of 21 and 25 showed phosphorylation potential of 0.997 and 0.993 respectively, threonine showed a phosphorylation potential of 0.923 at 283 , tyrosine showed a max potential of 0.938 at residue position 277. In gi_40142120 (Figure 2E) serine at 22 and 191 positions showed a phosphorylation potential of 0.992 and 0.991 respectively, threonine showed a phosphorylation potential of 0.959 at residue position of 28 while tyrosine showed a phosphorylation potential of 0.932 at residue position of 278. In gi_68126239 (Figure 2F) serine showed max phosphorylation potential of $0.996,0.992$ and 0.991 respectively at residue positions of 21, 25, 190, threonine showed max phosphorylation potential of 0.923 at residue position of 283 and tyrosine showed a max phosphorylation potential 0.932 at 277. In gi_68520370 (Figure 2G) serine showed phosphorylation potential of 0.997 and 0.993 at residue positions 21 and 25 respectively, similarly threonine showed phosphorylation potential of 0.923 at residue position of 283 where as tyrosine showed phosphorylation potential of 0.938 at residue position 277. In gi_73169547 (Figure 2H) serine showed max phosphorylation potential of 0.997 and 0.993 at residue positions 21 and 25, threonine showed max phosphorylation

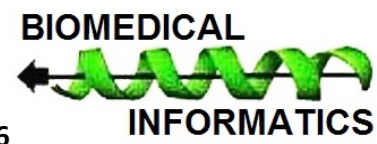




\section{Open access}

potential of 0.923 at residue position 283, tyrosine showed phosphorylation potential of 0.938 at residue position 277. In gi_73170759 (Figure 2I) serine showed phosphorylation potential of 0.997, 0.993, 0.993 at residue positions 482, 949 and 1057 respectively, threonine showed max phosphorylation potential of 0.892 at residue position 664, tyrosine showed max phosphorylation potential 0.809 at residue position 56. In gi_7658170 (Figure 2J) serine showed phosphorylation potential of 0.997 at residue position 22 whereas threonine and tyrosine didn't show any potential of phosphorylation.

\section{Conclusion:}

\section{References:}

[1] Alexander J \& Russell DG, Advan Parasitol. 1992 31: 175 [PMID: 1496927]

[2] Murray HW et al. Lancet 2005 366: 9496 [PMID: 16257344]

[3] Hanks SK, Ann Marie Quinn Meth Enzymol 1991200 [PMID: 1956325]

[4] Salotra P et al. International Journal of Biochemistry and Cell Biology 2000, 32 (1) 309

[5] Pereira RM et al. FASEB J. 2010 24(2) 617
The study reveals that serine, threonine and tyrosine residues of protein kinases having phosphorylation potential close to 1 could be an important target for anti-leishmanial therapies. Hence, different species strains of Leishmania provide important insights into development of anti-leishmanial drug regimes using kinase inhibitors.

\section{Acknowledgement:}

Sukrat Sinha is thankful to the financial support provided by the Department of Science and Technology (India) Fast Track scheme for Young Scientists No. SB/FT/LS-345/2012.

[PMID: 19812373]

[6] Vlachakis D et al. Peer J 2015 e725 [PMID: 25648563]

[7] Cardenas D et al. Mol Microbiol. 2015 96(1) [PMID: 25582232]

[8] Tsigankov et al. J Proteome Res. 2013 12(7) 3405 [PMID: 23688256]

[9] Tsigankov et al. Mol Cell Proteomics. 2014 13(7) 1787 [PMID: 24741111]

Edited by $\mathbf{P}$ Kangueane

Citation: Sinha \& Sundaram, Bioinformation 12(4): 249-253 (2016) License statement: This is an Open Access article which permits unrestricted use, distribution, and reproduction in any medium, provided the original work is properly credited. This is distributed under the terms of the Creative Commons Attribution 